\title{
A left temporal lobe impairment of auditory information processing in schizophrenia:
} An event-related potential study

\author{
U. Schall ${ }^{1,2}$, A. Schön ${ }^{1}$, D. Zerbin ${ }^{1,2}$, S. Bender ${ }^{2}$, C. Eggers ${ }^{1}$, R.D. Oades ${ }^{1}$ \\ University Clinic for Child and Adolescent Psychiatry (1) University Clinic for Adult Psychiatry (2), \\ (RLHK), Virchowstrasse 174, 45147 Essen, Germany
}

1997 Neuroscience Letters, 229, 25-28.

\begin{abstract}
:
A measure of auditory prepulse inhibition (PPI) is the reduction of the scalp-recorded P1 eventrelated potential (ERP) after a sound that is preceded by $100-300 \mathrm{~ms}$ by a click as prepulse. This measure of sensory gating was adapted to study the effect of a prepulse on processing tones that were part of a 'go no-go' discrimination.

ERPs were recorded at right and left, frontal and temporal sites in groups of patients with schizophrenia $(\mathrm{SCH})$ or obsessive compulsive disorder (OCD) and healthy controls (CON). A prepulse $100 \mathrm{~ms}$ but not $500 \mathrm{~ms}$ before either tone reduced the P1 ERP amplitude in healthy and OCD subjects but not $\mathrm{SCH}$ patients. At frontal and temporal recording sites the $\mathrm{P} 1$ amplitude was similar bilaterally in controls but showed a right temporal shift in the SCH patients. If the tone was the 'no-go' tone, the prepulse reduced the $\mathrm{N} 1$ amplitude in both the $\mathrm{CON}$ and $\mathrm{SCH}$ groups. The $\mathrm{N} 1$ was similar, bilaterally in controls but again showed a right temporal shift in the $\mathrm{SCH}$ group.

These results show a reduction of a PPI-like effect on early processing (P1) that is more marked in the left hemisphere of $\mathrm{SCH}$ patients and may affect channel selection for processing information (N1) about task-relevant sounds.
\end{abstract}

Keywords: Prepulse inhibition; Sensory gating; P1/P50; Frontal and temporal lobe; Schizophrenia; Lateralization

If a brief click precedes another by $100-$ $300 \mathrm{~ms}$, the response to the second (S2) is reduced by comparison with shorter or longer inter-stimulus intervals (ISIs). This phenomenon of prepulse inhibition (PPI) is a measure of sensory gating on the processing of S2. In one form of the test S2 is salient and the size of a startle response in animals or humans is recorded. A second test-form, more appropriate for studies in humans, uses two similar stimuli and an event-related potential (ERP) response is recorded, the P50 or P1 [6]. We developed a third form where S2 is part of a discrimination (target or non-target). This enables the recording of response in terms of signal detection and ERP measures of controlled information processing, rather than only automatic processes as in other test forms [17]. Thus, with a $100 \mathrm{~ms}$ ISI an increasingly liberal response bias depended on the increasing difficulty of the discrimination, but with $500 \mathrm{~ms}$ the response-bias did not differ from a no-prepulse control condition.

Animal studies emphasize the disruptive effects on PPI of too much mesolimbic serotonergic and dopaminergic activity and human work with ERPs emphasizes the nicotinic and noradrenergic systems in the hippocampal complex [6]. There are conflicts over the role of the frontal cortex and mesocortical dopamine in PPI of the startle response in animals $[2,19]$. But, damage to the human temporal lobe, that contains P1 generators, affects performance of both taskforms $[6,9,15]$.

As PPI is reported to be attenuated in schizophrenic patients $[1,6]$ and, to some extent, in patients with obsessive-compulsive disorder [18], its expression at frontal and temporal sites over the left and right hemisphere was studied with a method based on that of Braff et al. [1]. Further to the 
reasons advanced above, differences were expected between frontal and temporal sites as schizophrenics with deficits on card-sorting tests of frontal function show major PPI loss [3] and their decreased ERP amplitude has been related to atrophy in the left superior temporal gyrus [10]. Changes in left temporal ERPs associated with attentional function have been noted in schizophrenic patients [14]. We predicted that PPI in a discrimination testform might support the proposal of a temporal lobe functional and anatomical asymmetry, where a lack of PPI has been proposed to explain how trivial information might become the source of information for hallucinations and delusions [6].

There were 6 male and 2 female schizophrenic patients ( $\mathrm{SCH}$ ) diagnosed by two psychiatrists according to ICD 10 and DSM IIIR, receiving a mean dose of $415 \mathrm{mg}$ chlorpromazine equivalents of medication. They had a mean age of 22.1 years (SD 7, range 15-34), an IQ of 99 (SD 15, Raven's standard progressive matrices) and had spent 7-12 y at school. Seven males and three females with obsessive-compulsive disorder (OCD) showed no psychotic features, minimal symptoms of depression and no psychotropic medication. They had a mean age of 22.7y (SD 6 , range 16-33), an IQ of 119 (SD 13) and had spent $10-13 y$ at school. The 9 male and 10 female healthy controls (CON) reported no psychotropic medication, no major medical problems and no psychiatric illness for themselves or their first-degree relatives (mean age, 25.4y, SD 2.9 y, range 17-27y; IQ 123, SD 10). All were right-handed according to their writing preference and they or their parents gave informed, signed consent.

Tones were played bilaterally over DT48 headphones (Bayer Dynamics). The prepulse (S1) was an $0.6 \mathrm{~ms}$ alternating rectangular click at $50 \mathrm{dBSL}$ (adjusted by a calibrated sound pressure function), presented against white noise (30 dBSL). The second pulse (S2), after 100 or $500 \mathrm{~ms}(P=0.5)$, was a 0.8 or 1.4 $\mathrm{kHz}$ tone $(P=0.5)$ at $65 \mathrm{dBSL}$ lasting $50 \mathrm{~ms}$ (rise/fall time $10 \mathrm{~ms}$; gate-functiongenerator). Interval- and tone-types alternated in a Bernoulli sequence of 100 stimulus-pairs per block with an inter-pair interval of 4-6 s: (optimal ERP expressed at ca. $8 \mathrm{~s}$ intervals). Each subject received 4-6 trialblocks. Detection thresholds were determined before the test $(0.125-8 \mathrm{kHz}$; Audio-Med $B C A 3)$ and sound levels adjusted if the threshold varied by $.5 \mathrm{~dB}$. Subjects sat in a sound-attenuated, electrically-isolated room lit by a $25 \mathrm{~W}$ lamp. They were asked to discriminate between the tones and press a button with their dominant hand after the higher tone while fixating a cross $1.5 \mathrm{~m}$ in front of them to minimize eye-movements.

The EEG activity was recorded from 19 sites in an electrocap (international 10-20 system). Data were analyzed from F3/F4 and T3/T4 comparisons using a linked mastoid reference and Fp ground (all impedance's <2 $k \Omega$ ). The signal was amplified $12000^{\prime}$ (band pass $0.1-70 \mathrm{~Hz}$ ). Sweeps were rejected if the EOG exceeded $65 \mathrm{~V}$ from $2 \mathrm{~s}$ before $\mathrm{S} 1$ onset until $1 \mathrm{~s}$ after $\mathrm{S} 2$ onset (50-60\% of trials were rejected in the OCD and CON groups and 70$80 \%$ in the $\mathrm{SCH}$ group). Thus recordings free of horizontal or vertical movement artifacts were obtained (80-200: minimum of 20/ stimulusISI combination) and digitized at $250 \mathrm{~Hz}$ for 1300 ms after S1. Peaks were measured with reference to a $50 \mathrm{~ms}$ pre-S1-baseline. In the $500 \mathrm{~ms}$ condition the baseline was adjusted to the contingent negative variation $(0.9-2.4 \mathrm{mV})$ measured at S2-onset ${ }^{1}$. The time windows for identifying peaks after S2 were: P1 30-80 ms, $\mathrm{N} 1$ 81-140 ms, and P3 280-450 ms [12]. The parallel-test reliability for amplitude measures between blocks ranged $r=0.79-0.95$.

The analysis concentrates on the relative inhibition of the P1, N1 and P3 amplitude elicited by a target in the task induced by a prepulse $100 \mathrm{~ms}$ earlier vs. a $500 \mathrm{~ms}$ interval, at which the prepulse causes a negligible effect on the startle reflex or the P1 amplitude $[7,18]$. This measure of sensory gating has

\footnotetext{
${ }^{1}$ This correction did not contaminate measures of the effect of S1 on S2: (1) SCH patients had smaller N1 peaks after both stimuli than the other groups $F(2,32)=10.2-12.4, P<$ $0.0003)$, without an interaction with stimulus, (2) $\mathrm{N} 1$ amplitudes after S1 and S2 correlated within each group (Spearman rho $=0.65-0.89)$, (3) normalization of the data removed any confounding influence of individuals on group data across conditions.
} 
been used to show interactions with reaction time and performance (e.g. a significant regression for amplitude suppression with perceptual sensitivity, signal detection criterion and error rate $[17,18])$. Specifically, ERP peak measures were collapsed firstly across tones ('gating measures', 100 vs. $500 \mathrm{~ms}$ ) and secondly across ISI ('discrimination measures', target vs. non-target) and normalized to a scale from 0 to +1 (maximum-minimum method [11]). Separate MANOVAs for the P1, N1 and P3 peaks by recording site by group (with the Greenhouse-Geisser adjustment of the degrees of freedom) was followed by univariate ANOVA of the hemispheric dominance index (HDI; amplitude differences between the left and right hemispheres, at F3 minus F4, T3 minus T4) by group for the P1, $\mathrm{N} 1$, and $\mathrm{P} 3$ peaks in the 'gating' and 'discrimination' measure. The locus of the differences was examined post hoc with the Scheffe' test.

Within a window of $0.8 \mathrm{~s}$ after the target the patients showed a slower mean reaction time (SCH 509 SD 59 ms; OCD 509 SD 62 ms; CON 405 SD 34 ms; $F(2,38)=8.7, P<0.001)$ and more errors of omission than controls (SCH 15 SD 9\%; OCD 13 SD 4\%; CON 3 SD 1\%; $F$ $(2,38)=7.3, P<0.05)$. Neither measure correlated with IQ.

Figure 1, with the waveforms at $\mathrm{Cz}$ to facilitate comparison with other ERP reports of sensory gating [12], shows a larger P3 component after the non-target vs. target tone, as is usual for 'go no-go' tasks where the stimuli occur with a $50 \%$ probability [4]. A comparison of ISI conditions independent of tone-type showed the presence of PPI of the $\mathrm{P} 1$ component in CON at the shorter ISI (top two vs bottom two parts of Fig. 1, solid-line); this difference was not found in the $\mathrm{SCH}$ group (dotted waveform, $F(2,38)=3.3, P<0.05$ ). But the type of tone (target/non-target) did interact with ISI for the $\mathrm{N} 1$ in both the control $(F(3,57)=2.9 ; \mathrm{e}=0.88 ; P<0.05)$ and the $\mathrm{SCH}$ groups $(F(3,24)=3.7$; e $=0.77 ; P<0.05)$, showing a PPI-like effect after the non-target tone. Only after the non-target tone and only in controls was a PPI like effect evident in the P3 component $(F(3,57)=3.3 ; \mathrm{e}=0.91 ; P<$
0.05). No significant interactions of ISI with components were evident for the OCD subjects [18]. Thus a PPI-like effect was seen in the 100 vs 500 ms condition on N1 elicited by the non-target: this effect on the P3 was absent in both patient groups and, independent of tone, was absent for P1 in the $\mathrm{SCH}$ group.
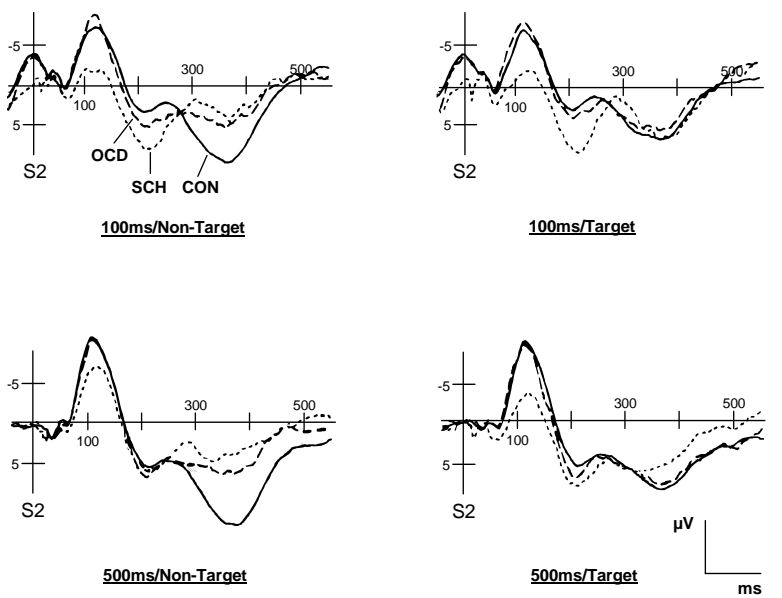

500ms/Non-Target

Fig. 1. Grand mean waveforms in an auditory discrimination task after non-target $(0.8 \mathrm{kHz})$ and target $(1.4 \mathrm{kHz})$ tones $(P=0.5)$ at $\mathrm{Cz}$ when a click preceded the tones by 100 or $500 \mathrm{~ms}(P=0.5)$. $\mathrm{CON}$, healthy controls (solid line); $\mathrm{SCH}$, schizophrenic patients (dotted line); OCD, obsessive-compulsive patients (dashed line).

Inspection of ERP components over each hemisphere showed that whereas $\mathrm{P} 3$ peaks were smaller in the patients $(\mathrm{T} 3 / \mathrm{T} 4,3.0$ $3.2 / 3.6$ vs $4.4 / 4.8 \mu \mathrm{V}$; $\mathrm{CON}$ ), for $\mathrm{SCH}$ patients N1 was smaller at frontal sites (F3/F4, -5.3/5.1 vs. $-7.9 /-7.6 \mu \mathrm{V} ; \mathrm{CON})$ and $\mathrm{P} 1$ larger at temporal sites than in OCD and CON groups (T3/T4, $+0.8 /+1.0$ vs. -0.3 to $+0.1 /-0.1$ to +0.3 $\mu \mathrm{V}$; CON and OCD; Scheffe test, $P<0.05)$. The analysis proceeded with normed data to accommodate for inhomogeneity in the raw data and multiplicative effects on ERPs of changes of source strength in ANOVA [11]. Normed ERP amplitudes in Fig. 2 compare the target vs non-target (collapsed across ISI) with the PPI condition (collapsed across tone-type).

Collapsed across tone-type, PPI of the P1 and P3 components did not differ across frontal and temporal sites for controls $(F$ $(3,57)=2.6 ; P=0.11$ and $F(3,57)=0.7 ; P=$ 0.43 , respectively): but a significant main effect was found for PPI of the P1 peak across groups $(F(3,99)=4.3 ; \mathrm{e}=0.71 ; P<0.05$; Fig. 
3). Post hoc analyses confirmed the presence of PPI on the P1 component only in the CON group (CON. SCH at F3, F4 and T3; CON. OCD at F4; Scheffe test, $P<0.05)$. The significant site by group interaction $(F(6,99)=2.6$; e = $0.71 ; P<0.05)$ was due to a positive shift at the right-temporal site in $\mathrm{SCH}(F(1,25)=8.6 ; P$ $<0.01)$. The other two groups did not show this lateralization (Fig. 3, bottom right vs. left). Consideration of the P1 amplitude after target and non-target tones, collapsed across ISI, yielded no differences between sites within the controls nor between groups.

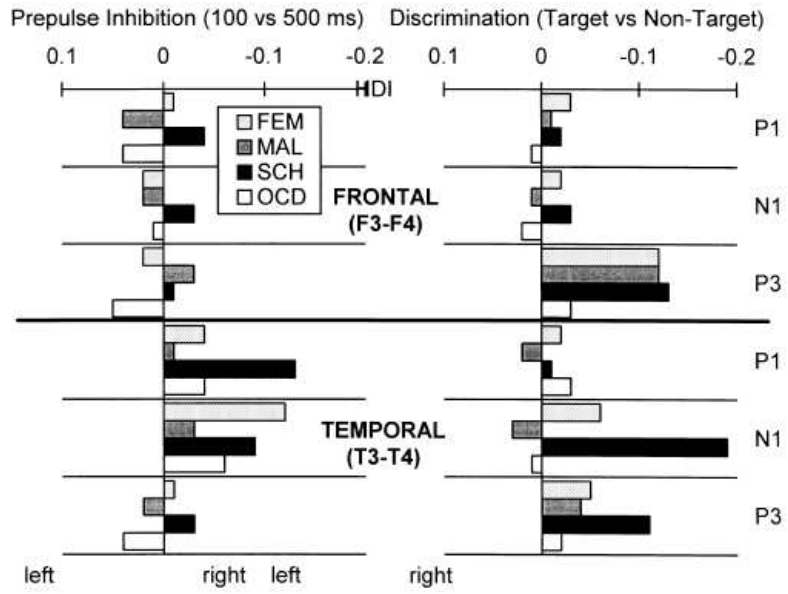

Fig. 2. Hemispheric dominance indices (HDI; left minus right side) for PPI measures (normalized ERP amplitudes, 100 minus $500 \mathrm{~ms}$ ISI, left) and discrimination measures (target minus non-target normalized ERP amplitudes, right) for healthy controls (CON, separately for females and males), patients with schizophrenia $(\mathrm{SCH})$ or with obsessive-compulsive disorder (OCD). Negative values show a relative dominance of the right hemisphere (e.g. SCH group).

Collapsed across tone-type, PPI of the N1 component differed across frontal and temporal sites for controls. It was larger on the right, at T4 $(F(3,57)=6.4 ; \mathrm{e}=0.68 ; P<$ 0.01; Fig. 2, bottom left). But collapsed across ISI the N1 component did not vary between sites in the control group $(F(3,57)=2.2 ; P=$ $0.1)$. However, in this discrimination condition the topography did differ between groups $(F(3,99)=3.04 ; \mathrm{e}=0.75 ; P<0.05)$; there was an interaction with group $(F(6,99)=3.6$; $\mathrm{e}=$ $0.75 ; P<0.025)$ as a result of a lateralization over temporal sites (T3 vs. T4; $F(2,33)=7.6 ; P$ $=0.002)$. This effect was due to a right hemispheric shift in the $\mathrm{SCH}$ group $(\mathrm{SCH}-\mathrm{HDI}=$ $-19.3 \%$, SD 8.9 vs. CON-HDI $=-2.9 \%$, SD 7.5; $F(1,25)=11.9 ; P=0.002$; Fig. 2, bottom right).
F3

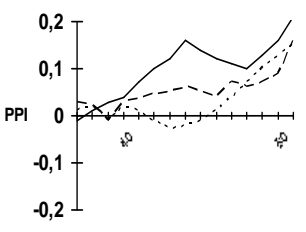

$\underline{T 3}$

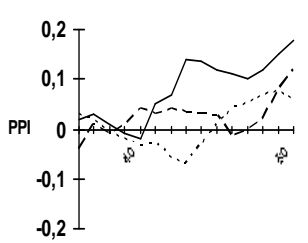

$\underline{\text { F4 }}$

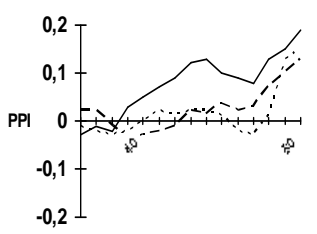

T4

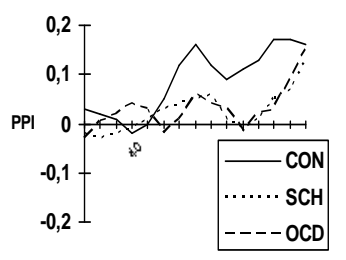

Fig. 3. Difference waveforms (500 minus $100 \mathrm{~ms}$ prepulse interval); Group means for normalized data (NU, normalized unit, scale 0 to \pm 1 ) over 24$80 \mathrm{~ms}$ after S2 (collapsed across tone-type) at F3, F4, T3, and T4 recording. Only the healthy controls (CON) showed significant PPI of the P1 amplitude.

The P3 amplitude after target and nontarget tones was enhanced on the right at frontal sites in controls $(\operatorname{CON} F(3,57)=7.7$; $\mathrm{e}=$ $0.74 ; P<0.01)$ and this was confirmed across groups $(F(3,99)=5.4 ; \mathrm{e}=0.75 ; P<0.01)$ with no significant variation $(F(6,99)=1.04 ; P=$ $0.4)$.

Thus a quiet click 100 vs. one $500 \mathrm{~ms}$ before a tone, can reduce the expression of $\mathrm{P} 1$ and N1 ERPs recorded over the left temporal hemisphere of $\mathrm{SCH}$ patients compared to records from the right side or from either side in controls. This effect is evident for the N1 potential if the eliciting tone is the 'no-go' stimulus in discrimination, but the effect is seen for the P1 irrespective of tone-type.

Considering that sources for both ERPs are reported from structures in the temporal lobe [15], the differential effect on the two peaks is consistent with two early stages of processing auditory information. Thus, P1 may represent the registration of the arrival of sound information in the auditory cortex, while the $\mathrm{N} 1$ may represent the subsequent allocation of channels for further processing when the stimulus has significance, as here in a twotone discrimination $[8,9,13]$.

A lateralization of ERP responses in a PPI paradigm using a test with just one soundtype has not been reported [12]. However, an 
impairment of early information processing over the left temporal lobe of $\mathrm{SCH}$ patients, and not OCD or healthy subjects, is consistent with reports of asymmetry of later ERP indices of auditory processing and in the potential temporal lobe substrates $[5,10]$. ERPs sensitive to stimulus deviance with similar early latencies have been recorded from temporal lobe structures such as the hippocampus in animals [16] and the mismatch negativity response to stimulus deviance is disrupted using tones presented at similar ISIs [20]. Mismatch negativity is also reported to have a source in the auditory temporal cortex in humans and is recorded from more lateral and temporal sites in $\mathrm{SCH}$ patients than in healthy subjects [13]. It is now of interest to examine the role of neuroleptic medications on lateralized information processing in these patients, who are reported to show an imbalance of mesocortical catecholamine function.

We thank Rita Frantska, Jutta Haverkorn, Mark Pearson, and Robert Windelschmitt for technical assistance, Dr. Philip Ward for discussion and the German Research Council (DFG Scha 626/1-1) for financial support.

\section{References:}

[1] Braff, D.L., Grillon, C. and Geyer, M.A., Gating and habituation of the startle reflex in schizophrenic patients, Arch. Gen. Psychiatry, 49 (1992) 206-215.

[2] Bubser, M. and Koch, M., Prepulse inhibition of the acoustic startle response of rats is reduced by 6 -hydroxydopamine lesions of the medial prefrontal cortex, Psychopharmacology, 113 (1994) 487-492.

[3] Butler, R.W., Jenkins, M.A., Geyer, M.A. and Braff, D.L., Wisconsin card-sorting deficits and diminished sensorimotor gating in a discrete subgroup of schizophrenic patients. In C. A. Tamminga and S. C. Schulz (Eds.), Schizophrenia, Raven Press, New York, 1990.

[4] Czigler, I., Csibra, G. and Ambro, A., Aging, stimulus identification and the effect of probability: an event-related potential study, Biol. Psychol., 43 (1996) 27-40.

[5] Egan, M.F., Duncan, C.C., Suddath, R.L., Kirch, D.G., Mirsky, A.F. and Wyatt, R.J., Eventrelated potential abnormalities correlate with structural alteration and clinical features in patients with chronic schizophrenia, Schizophr. Res., 11 (1994) 259-271.

[6] Freedman, R., Adler, L.E., Bickford, P., Byerley, W., Coon, H., Cullum, C.M., Griffith, J.M., Harris, J.G., Leonard, S.S., Miller, C., MylesWorsley, M., Nagamoto, H.T., Rose, G. and Waldo, M., Schizophrenia and nicotinic receptors, Harvard Rev. Psychiatry, 2 (1994) 179-192.

[7] Guterman, Y., Josiassen, R.C. and Bashore, T.R., Attentional influence on the P50 component of the auditory event-related brain potential, Int. J. Psychophysiol., 12 (1992) 197-209.

[8] Hansen, J.C. and Hillyard, S.A., Selective attention to multidimensional auditory stimuli, J. Exp. Psychol. (Hum. Percept. Perform.), 9 (1983) 1-19.

[9] Knight, R.T., Scabini, D., Woods, D.L. and Clayworth, C., The effects of lesions of superior temporal gyrus and inferior parietal lobe on temporal and vertex components of the human AEP, Electroenceph. Clin. Neurophysiol., 70 (1988) 497-509.

[10] McCarley, R.W., Shenton, M.E., O'Donnell, B.F., Faux, S.F., Kikinis, R., Nestor, P.G. and Jolesz, F.A., Auditory P300 abnormalities and left superior temporal gyrus volume reduction in schizophrenia, Arch. Gen. Psychiatry, 50 (1993) 190-197.

[11] McCarthy, G. and Wood, C., Scalp distributions of event-related potentials: an ambiguity associated with analysis of variance models, Electroenceph. Clin. Neurophysiol., 62 (1985) 203-208.

[12] Nagamoto, H.T., Adler, L.E., Waldo, M.C., Griffith, J. and Freedman, R., Gating of auditory response in schizophrenics and normal controls, effects of recording site and stimulation interval on the P50 wave, Schizophr. Res., 4 (1991) 31-40.

[13] Oades, R.D., Connections between studies of the neurobiology of attention, psychotic processes and event-related potentials. In G. Karmos, M. Molnar, I. Czigler and J.E. Desmedt (Eds.), Perspectives of Event-Related Potentials Research, Electroenceph. Clin. Neurophysiol., Suppl. 44 (1995) 428-43.

[14] Oades, R.D., Zerbin, D. and Eggers, C., Negative difference ( $\mathrm{Nd}$ ), an ERP marker of stimulus relevance: different lateral asymmetries for paranoid and nonparanoid 
schizophrenics, Pharmacopsychiatry, 27 (1994) 65-67.

[15] Reite, M., Teale, P., Goldstein, L., Whalen, P. and Linnville, S., Late auditory magnetic sources may differ in the left hemisphere of schizophrenic patients: a preliminary report, Arch. Gen. Psychiatry, 46 (1989) 565-572.

[16] Ruusuvirta, T., Korhonen, T., Penttonen, M. and Arikoski, J., Hippocampal evoked potentials to pitch deviances in an auditory oddball situation in the rabbit: no human mismatch-like dependence on standard stimuli, Neurosci. Lett., 185 (1995) 123-126.

[17] Schall, U. and Ward, P.B., Prepulse inhibition facilitates a liberal response bias in an auditory discrimination, NeuroReport, 7 (1996) 652-656.

[18] Schall, U., Schön, A., Zerbin, D., Eggers, C. and Oades, R.D., Event related potentials during an auditory discrimination with prepulse inhibition in patients with schizophrenia, obsessive-compulsive disorder and healthy subjects, Int. J. Neurosci., 84 (1996) 15-33.

[19] Swerdlow, N.R., Braff, D.L., Geyer, M.A. and Koob, G.F., Central dopamine activity in rat mimics abnormal acoustic startle response in schizophrenics, Biol. Psychiatry, 21 (1986) 2333.

[20] Winkler, I. and Näätänen, R., Event-related potentials in auditory backward recognition masking: a new way to study the neurophysiological basis of sensory memory in humans, Neurosci. Lett., 140 (1992) 239-242. 